\title{
Assessment of endoscopic sinus surgery in management of sinonasal diseases
}

\author{
Sayed Attia Siam, Mohammed Kamel Elawady, \\ Omer Mohammed Elemery \\ Otorhinolaryngology and Head and Neck Surgery Department, Faculty of Medicine, Al-Azhar \\ University \\ Corresponding author: Omer Mohammed Elemery, email: omerelemery@gmail.com
}

\begin{abstract}
Background: functional endoscopic sinus surgery (FESS) has revolutionized surgical care, opening new horizons in the management of chronic rhinosinusitis and other paranasal sinus disorders Messerklinger established and reinterated the importance of the sinus ventilation and pattern of mucociliary clearance. FESS was first described independently by both Messerklinger in German literature and Wigand.

Aim of the work: this study aimed to assess the efficacy, safety and benefits of FESS in cases of chronic recurrent rhinosinusitis with or without nasal polyposis, fungal sinusitis,septal and turbinate pathology and CSF rhinorhea in terms of morbidity, mortality and recurrent of disease. Patients and Methods: this study was conducted in Bab Elshaerea University Hospital and Hearing and Speech Institute from July 2017 to August 2018. A total of 50 patients with clinical evidence of sinonasal diseases were categorized into 4 groups (Chronic recurrent rhinosinusitis with or without nasal polyposis (20 patients), fungal sinusitis (10 patients),septal and turbinate pathology(10 patients) and CSF rhinorhea(10 patients)). Patients were evaluated with nasal endoscopy and computed tomographic (CT) evaluation prior to FESS. Results: out of 50 patients, 26 were male and 24 were female in the present study. Male: female ratio was 52:48. Depending on symptoms, endoscopic examination and CT scanning, three patients only had recurrence (Two patients from group of chronic recurrent rhinosinusitis with or without nasal polyposis and one patient from fungal sinusitis group). Conclusion: FESS provided an excellent and safe method for treating sinonasal disease. The success rates were encouraging, but because of the nature and chronicity of the disease, longer follow-up was necessary to truly assess the surgical effectiveness of the procedure.
\end{abstract}

Key words: surgery, sinonasal disease.

\section{Introduction}

Functional endoscopic sinus surgery is a complex procedure used by otorhinolaryngologists to treat a host of nasal sinus pathologies (1). Functional endoscopic sinus surgery (FESS) is a minimally invasive surgical treatment which uses nasal endoscopes to enlarge the nasal drainage pathways of the paranasal sinuses to improve sinus ventilation ${ }^{(2)}$.This procedure is generally used to treat inflammatory and infectious sinus diseases, including chronic rhinosinusitis that doesn't respond to drugs, nasal polyps, some cancers, and decompression of eye sockets/optic nerve in Graves ophthalmopathy ${ }^{(3-5)}$. The first recorded instance of endoscopy being used for visualization of the nasal passage was in Berlin, Germany in $1901^{(6)}$. Alfred Hirschmann, who designed and made medical instruments, modified a cytoscope to be used in the nasal cavity. Hirschmann published Endoscopy of the nose and its accessory sinuses ${ }^{(7)}$. Reichart performed the first endoscopic sinus surgery using a $7 \mathrm{~mm}$ endoscope. Maltz also encouraged the use of endoscopes as a diagnostic tool for nasal and sinus abnormalities (6). 
Harold Hopkins used his background in physics to develop an endoscope that provided more light and had drastically better resolution than previous endoscopes (6). Walter Messerklinger published the book titled Endoscopy of the Nose on his findings and his proposed methods to utilize nasal endoscopy for diagnosis (8). After learning of Messenklinger's endoscopic techniques, David Kennedy and Karl Storz developed instruments for use in endoscopic sinus surgery, and coined the term Functional Endoscopic Sinus Surgery ${ }^{(9)}$.Kennedy published multiple papers on FESS use and technique, and in 1985 the first course on FESS was taught in Johns Hopkins Medical Center ${ }^{(6)}$.

\section{Aim of the work}

This study aimed to assess the efficacy, safety and benefits of FESS in cases of chronic recurrent rhinosinusitis with or without nasal polyposis ,fungal sinusitis,septal and turbinate pathology and CSF rhinorhea in terms of morbidity, mortality and recurrent of disease.

\section{Materials and Methods}

This study was conducted in the Department of Otorhinolaryngology and Head and Neck Surgeryat Bab El-Shaerea Hospitals and Hearing and speech institute. A total of 50 patients categorized into 4 groups (Chronic recurrent rhinosinusitis with or without nasal polyposis (20 patients) ,fungal sinusitis (10 patients), septal and turbinate pathology(10 patients) and CSF rhinorhea(10 patients)), who were evaluated with nasal endoscopy and computed tomographic evaluation prior to FESS. These cases were selected from the patients attending the ear nose and throat (ENT) outpatient department (OPD). All patients in this studied group were subjected to a detailed history of a wide spectrum of presenting symptoms viz. facial pain, headache, nasal discharge (Whether it is watery, mucoid, purulent or blood mixed), nasal obstruction (Its duration, whether it was continuous or intermittent and whether it is associated with any external nasal deformity). The presence of other symptoms, such as postnasal discharge, sneezing, acute/chronic/serous otitis media, was also noted in full details. The complete personal, past, and family history were also elicited in addition with past medical/surgical history to know about any chronic use of antihistaminic, steroid sprays, and other medications in the past. All patients were subjected to thorough ENT examination with special emphasis on anterior and posterior rhinoscopy. Nasal Endoscopy was done using Hopkins rod endoscopes $\left(0^{\circ}, 30^{\circ}\right)$ computed tomography (CT) of paranasal sinuses was done and CT cysternography was done in patients with CSF rhinorhea. After a detailed nasal endoscopy and CT-scan study, patients underwent surgery-FESS. The patients included in the present study were explained in details about alternative modes of treatment, nature of the surgery, outcomes of surgery including benefits as well as possible complications of surgery. They were also detailed with the need for regular post-operative follow-up to monitor healing and avoid post-operative complications.

The operative technique used was planned in accordance to the need of the individual case. Surgical endoscopic management of concha bullosa and surgery of deviated nasal septum (Endoscopic septoplasty) was always planned in concert with the treatment of inflammatory disease in adjacent osteomeatal complex, ethmoid, and maxillary sinus. In the case of presence of extensive inflammatory disease in ethmoids and maxillary sinus, coherent FESS was done after endoscopic excision of concha bullosa was carried out. In all the patient's concepts of the "Messerklinger technique" of FESS were followed. Postoperative medication included an oral course of broad spectrum antibiotic, analgesics, and antihistaminic.The medial orbital wall was skeletonized early in the dissection to provide an essential landmark and the lateral limit of the dissection. The second critical landmark, the skull base was usually identified in the posterior ethmoid. After entering the posterior ethmoid, the roof was slowly skeletonized by removing intercellular partitions working from posterior to anterior and staying close to the medial orbital wall. When the sphenoid 
sinus needed to be opened this was typically performed by infracturing the sphenoid bulge in the inferior and medial aspect of the posterior ethmoid or through sphenoethmoidal recess. The opening was then enlarged taking care to enlarge it inferiorly and medially to include the natural ostium of the sphenoid sinus. When the ethmoid roof was carefully identified, the dissection was then continued from posterior to anterior, following the slope of the ethmoid roof into the frontal recess. The zero degree $4 \mathrm{~mm}$ telescope was frequently changed for a $4 \mathrm{~mm} 30$ degree telescope. The anterior ethmoidal artery was sometimes found immediately inferior to the skull base just posterior to the ethmoid dome. Approximately at the level of a superior extension of the anterior wall of the ethmoid bulla the frontal sinus opening was usually found medially in close proximity to the middle tubrbinate. Care was taken to ensure that the natural ostium of the frontal sinus was identified. If the frontal sinus ostium was closed or markedly stenotic, it was enlarged. Care was taken to remove only the bone when possible and to disturb the mucosa as little as possible. Any granulation tissue or polyps were removed very cautiously. In limited disease, mucosa had been opened. When diffuse disease was present, total ethmoidectomy was continued until all disease identified by CT had been exenterated or marsupialized and ethmoidal cells with normal endoscopic ethmoidectomy, sphenoidotomy, antrostomy and meticulous dissection of the frontal recess was performed.

In cases with CSF rhinorhea, The defects varied, were in the roof of posterior ethmoids, cribriform plate and fovea ethmoidalis. It was necessary to open the ethmoids and to expose the skull base. After detection of the dehiscent area, the repair was done by grafts from mucosa and bone of middle turbinate or septal and mucoperichondrial grafts. The graft was positioned with gelfoam and surgicel. The cavity was packed for 2 weeks with prophylactic antibiotics. All patients were seen on a weekly basis in OPD until the turbinate and cavity healed completely. At each visit, local care consisted of suction of surgical cavities to remove discharge, clots, crusts to prevent synechiae formation between the middle turbinate and lateral nasal wall. If any adhesions were formed, they were released. The study was approved by the Ethics Board of AlAzhar University.

\section{Results}

\section{Chronic rhinosinusitis with and without polyps:}

Clinical Data: the results of 20 patients with chronic rhinosinusitis with and without polyps were analyzed. Age: the mean age was 39.55 years with a range of 18-55 years.

Sex: male: female ratio was $40: 60 \%$.

\section{Post-operative subjective assessment:}

Improvement was $90 \%$ (18 patients) with no improvement in $10 \%$ (2 patients).

One case presented by postoperative purulent sinus ostia and polypoid mucosa which was male patient aged 20 years old with dextrocardia and recurrent chest infection which was diagnosed by (kartagener syndrome) .Another case presented by nasal polyps and discharge which female patient was 18 years old with bronchial asthma and aspirin hypersensitivity (Samter's triad) which has high recurrence rate.

Table 1: showing post-operative subjective assessment of chronic sinusitis group

\begin{tabular}{|r|r|r|r|r|r|r|}
\hline & $\begin{array}{r}\text { Chronic sinusitis with } \\
\text { polyposis (10 patients). }\end{array}$ & $\begin{array}{r}\text { Chronic sinusitis without } \\
\text { polyposis (10 patients). }\end{array}$ & \multicolumn{2}{|}{$\begin{array}{r}\text { Total } \\
(20)\end{array}$} \\
\cline { 2 - 7 } & $\mathrm{N}$ & $\%$ & $\mathrm{~N}$ & $\%$ & $\mathrm{~N}$ & $\%$ \\
\hline No improvement(n=2) & 2 & $20 \%$ & - & - & 2 & $10 \%$ \\
\hline improvement $(\mathrm{n}=18)$ & 8 & $80 \%$ & 10 & $100 \%$ & 18 & $90 \%$ \\
\hline \hline
\end{tabular}

\section{Post-operative objective assessment:}

Endoscopic: the presence of polyp, discharge, edema, adhesions and scarring were considered as abnormal pathological findings. Normal cavities were detected in $65 \%$ 
Table 2: post-operative endoscopic examination of chronic sinusitis group with and without polyps

\begin{tabular}{|l|l|l|l|l|l|l|}
\hline \multirow{2}{*}{$\begin{array}{l}\text { Post-operative } \\
\text { Endoscopic } \\
\text { finding }\end{array}$} & \multicolumn{2}{|l|}{ Non polyposis (10 cases) } & \multicolumn{2}{l|}{$\begin{array}{l}\text { Middle meatal polyposis } \\
(4 \text { cases })\end{array}$} & \multicolumn{2}{l|}{$\begin{array}{l}\text { Diffuse polyposis } \\
(6 \text { cases })\end{array}$} \\
\cline { 2 - 7 } & $\mathrm{N}$ & $\%$ & $\mathrm{~N}$ & $\%$ & $\mathrm{~N}$ & $\%$ \\
\hline Normal (n=13) & 9 & $90 \%$ & 2 & $50 \%$ & 2 & $33.3 \%$ \\
\hline $\begin{array}{l}\text { Polyp and } \\
\text { discharge (n=1) }\end{array}$ & - & - & - & - & 1 & $16.7 \%$ \\
\hline $\begin{array}{l}\text { Purulent ostia } \\
(\mathrm{n}=1)\end{array}$ & - & - & - & - & 1 & $16.7 \%$ \\
\hline Crusts (n=5) & 1 & $10 \%$ & 2 & $50 \%$ & 2 & $33.3 \%$ \\
\hline
\end{tabular}

Radiologic: there were extermely significant improvements when comparing preoperative and postoperative radiological scores. When comparing preoperative and postoperative $\mathrm{CT}$ according to the extent of disease we found that there was extremely significant correlation. In postoperative CT sinus sinus opacity was detected in two cases one of them with polyps.According to lund Mackey's score one case has postoperative score 9 (kartegner's syndrome which had preoperative score 20) and other case has score 20 (Samter's triade which had preoperative score 20)

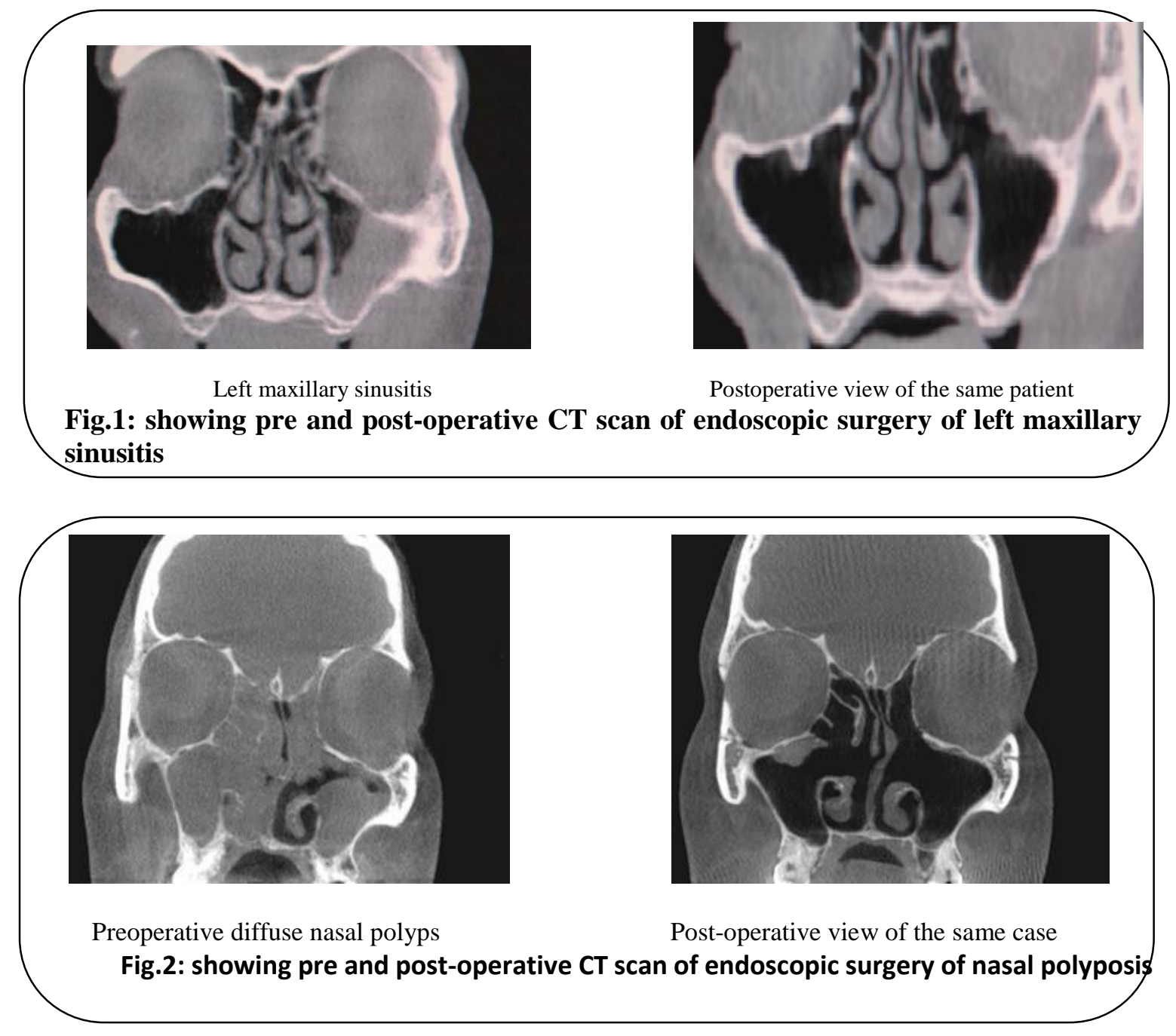

Complications: only one patient had intraoperative complication which is injury to lamina papyracea. Postoperative complications were reported in $20 \%$ of cases, one case had recurrent 
sinusitis and 2 cases had severe bleeding after removing nasal packs (no blood transfusion) and one case presented by recurrent polyposis.

Table 3: complications of endscopic surgery in chronic sinusitis group

\begin{tabular}{|l|l|l|}
\hline Intraoperative complications & No. of patients & Percentage (\%) \\
\hline $\begin{array}{l}\text { 1) No complications } \\
\text { a) Injury to lamina papyracea }\end{array}$ & 19 & $95 \%$ \\
\hline Postoperative complications: & 1 & $5 \%$ \\
\hline $\begin{array}{l}\text { 1)No complications } \\
\text { 2) Complicaitons: }\end{array}$ & 16 & $80 \%$ \\
a) Bleeding after removing nasal packs & 2 & $10 \%$ \\
b) Recurrent sinusitis & 1 & $5 \%$ \\
c) Recurrent polyposis & 1 & $5 \%$ \\
\hline
\end{tabular}

\section{Endoscopic treatment of fungal sinusitis}

Clinical Data: the results of 10 patients with fungal sinusitis were analyzed.

Age: the mean age was 38 years with a range (29-49 years).

Sex: male: female ratio was $30: 70 \%$

Post-operative subjective assessment:

Improvement $90 \%$ and no improvement in $10 \%$ (1 patient)

No improvement in one case that was bilateral fungal sinusitis the nose was not complete open and olfactory disturbance and discharge still present and progressive coarse worthily

Table 4: showing post-operative subjective assessment in fungal sinusitis group

\begin{tabular}{|c|c|c|c|c|c|c|}
\hline & & $\begin{array}{r}\text { Fungal ball } \\
\text { (7) }\end{array}$ & Allergic & $\begin{array}{r}\text { fungal } \\
\text { inusitis } \\
(3) \\
\end{array}$ & & $\begin{array}{r}\text { Total } \\
(10)\end{array}$ \\
\hline & $\mathrm{N}$ & $\%$ & $\mathrm{~N}$ & $\%$ & $\mathrm{~N}$ & $\%$ \\
\hline No improvement $(n=1)$ & -1 & - & 1 & $10 \%$ & 1 & $10 \%$ \\
\hline improvement $(n=9)$ & 7 & $77.8 \%$ & 2 & $22.2 \%$ & 9 & $100 \%$ \\
\hline
\end{tabular}

\section{Post-operative objective assessment:}

Endoscopic: the presence of polyp, discharge, edema, adhesions and scarring were considered as abnormal pathological findings. Normal cavities were detected in $80 \%$. One case presented by edematous inflamed mucosa and multiple polyps with thick viscous secretion described as (peanut butter) which present bilaterally but polyp present in right side only.

Table 5: post-operative endoscopic examination of fungal sinusitis

\begin{tabular}{|l|l|l|l|l|}
\hline \multirow{2}{*}{ Postoperative Endoscopic finding } & \multicolumn{2}{|l|}{ Fungal ball (7 cases) } & \multicolumn{2}{l|}{$\begin{array}{l}\text { Allergic fungal sinusitis } \\
\text { (3cases) }\end{array}$} \\
\cline { 2 - 5 } & $\mathrm{N}$ & $\%$ & $\mathrm{~N}$ & $\%$ \\
\hline Normal (n=8) & 7 & $70 \%$ & 1 & $10 \%$ \\
\hline $\begin{array}{l}\text { edematous inflamed mucosa and multiple } \\
\text { polyps with thick viscous secretion ( } \mathrm{n}=1)\end{array}$ & - & - & 1 & $10 \%$ \\
\hline Crusts $(\mathrm{n}=1)$ & - & - & 1 & $10 \%$ \\
\hline
\end{tabular}

Radiologic: there were extremely significant improvements when comparing preoperative and Postoperative radiological scores. When comparing preoperative and postoperative CT according to the extent of disease we found that, there was extremely significant correlation. In postoperative CT, sinus opacity was detected in one case which was bilateral fungal sinusitis with preoperative score was 18 . 


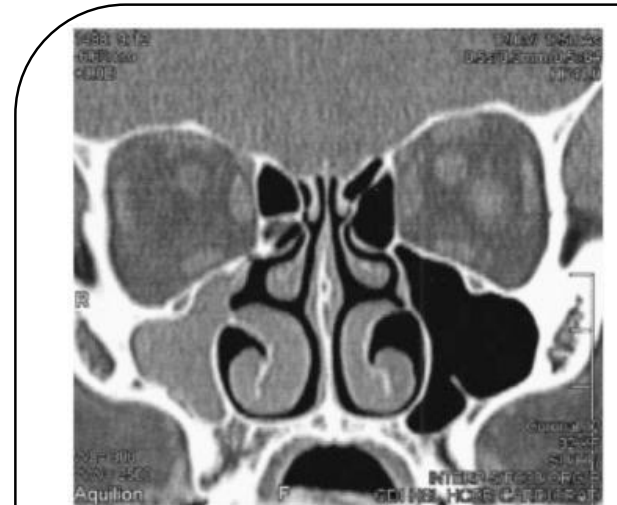

Right maxillary fungal ball

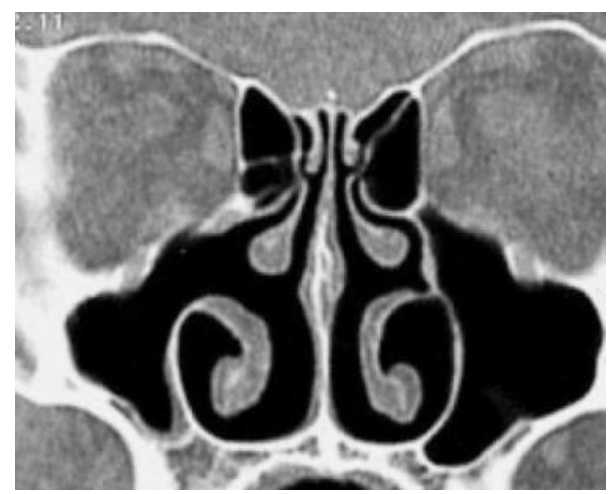

postoperative view

Fig.3: showing pre and post-operative CT scan of endoscopic surgery of right maxillary fungal ball

Complications: only recurrence in one case was a complication in this group who had isolated recurrent right sided frontal fungal sinusitis displaced the orbit downward and anterior in female patient 30 years old, endoscopic sinus surgery was done by using draf II b approach.

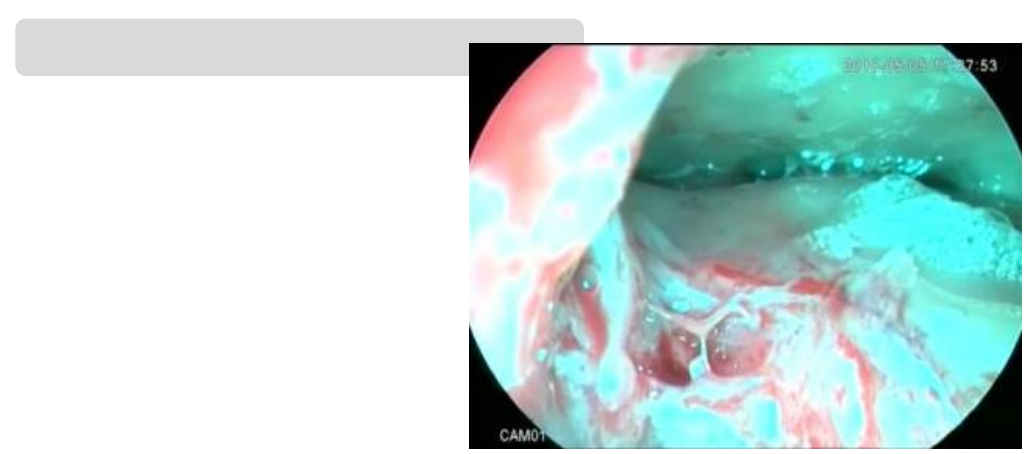

Fig.4: endoscopic view after draf II b in recurrent frontal fungal sinusitis

\section{Septal and turbinate pathology}

Clinical Data: the results of 10 patients with septal and turbinate pathology were analysed.

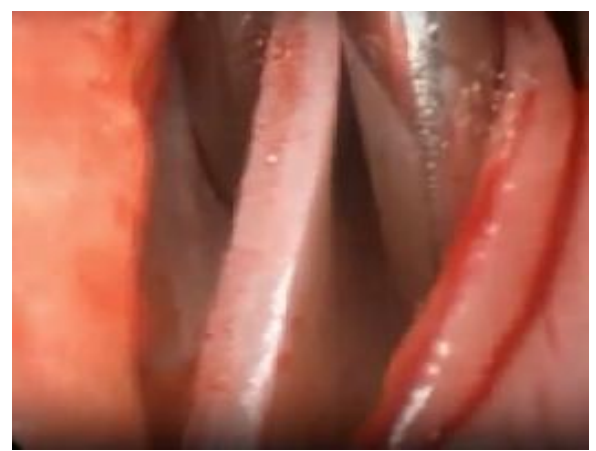

Fig.5: showing endoscopic septoplasty

Age: the mean age was 25 years with a range 17-32 years.

Sex: male: female ratio was 40: $60 \%$.

Marked improvement was detected in $90 \%$ and no improvement in $10 \%$ (1 patient).

Endoscopic: the presence of polyp, discharge, edema, adhesions or crusting and scarring were considered as abnormal pathological findings. Normal cavities were detected in $80 \%$. One case presented by left side adhesion near middle meatus. 
Table 6: post-operative endoscopic examination of septal and turbinate pathology group

\begin{tabular}{|c|c|c|c|c|c|c|c|c|}
\hline \multirow[t]{2}{*}{$\begin{array}{l}\text { Postoperative } \\
\text { Endoscopic } \\
\text { finding }\end{array}$} & \multicolumn{2}{|c|}{$\begin{array}{l}\text { Deviated nasal septum with } \\
\text { hypertrophied inferior } \\
\text { turbinate }(n=4)\end{array}$} & \multicolumn{2}{|c|}{$\begin{array}{l}\text { Deviated nasal } \\
\text { septum with } \\
\text { hypertrophied } \\
\text { inferior turbinate and } \\
\text { concha bellusa }(\mathrm{n}=2)\end{array}$} & \multicolumn{2}{|c|}{$\begin{array}{ll}\text { Hypertrophied } & \text { inferior } \\
\text { turbinates }(n=3) & \end{array}$} & \multicolumn{2}{|c|}{$\begin{array}{ll}\text { Hypertrophied } & \text { inferior } \\
\text { turbinates with } & \text { concha } \\
\text { bellusa }(\mathrm{n}=1) & \end{array}$} \\
\hline & No & $\%$ & No & $\%$ & No & $\%$ & No & $\%$ \\
\hline No $(n=8)$ & 2 & $50 \%$ & 1 & $50 \%$ & 3 & $100 \%$ & 1 & $100 \%$ \\
\hline Crusts $(n=1)$ & 1 & $25 \%$ & 1 & $50 \%$ & - & - & & \\
\hline $\begin{array}{l}\text { Adhesion, crusts } \\
\text { and scars }(n=1)\end{array}$ & 1 & $25 \%$ & - & - & - & - & & \\
\hline
\end{tabular}

Complications: adhesion was present in one case . Bleeding after removing of nasal packs occurred in one case (no blood transfusion).

Table 7: postoperative complications

\begin{tabular}{|l|l|l|}
\hline Postoperative complications: & & \\
\hline 1)No complications & 8 & $80 \%$ \\
2) Complications: & 1 & $10 \%$ \\
a) Bleeding after removing nasal packs & 1 & $10 \%$ \\
b) Nasal adhesions near middle meatus & $10 \%$ \\
\hline
\end{tabular}

\section{Endoscopic repair of spontaneous CSF rhinorhea}

10 cases of spontaneous CSF fistula were done, their ages ranged from 31 - 50 years, the mean age was 39 with male and female ratio is 40:60. The main complaint is watery nasal discharge were evident in CT scan cysternography.

All the patients had successful cessation of rhinorhea after a single procedure with postoperative leak free periods ranging from 6 months to One year. No major complications were occurred secondary to surgical management.
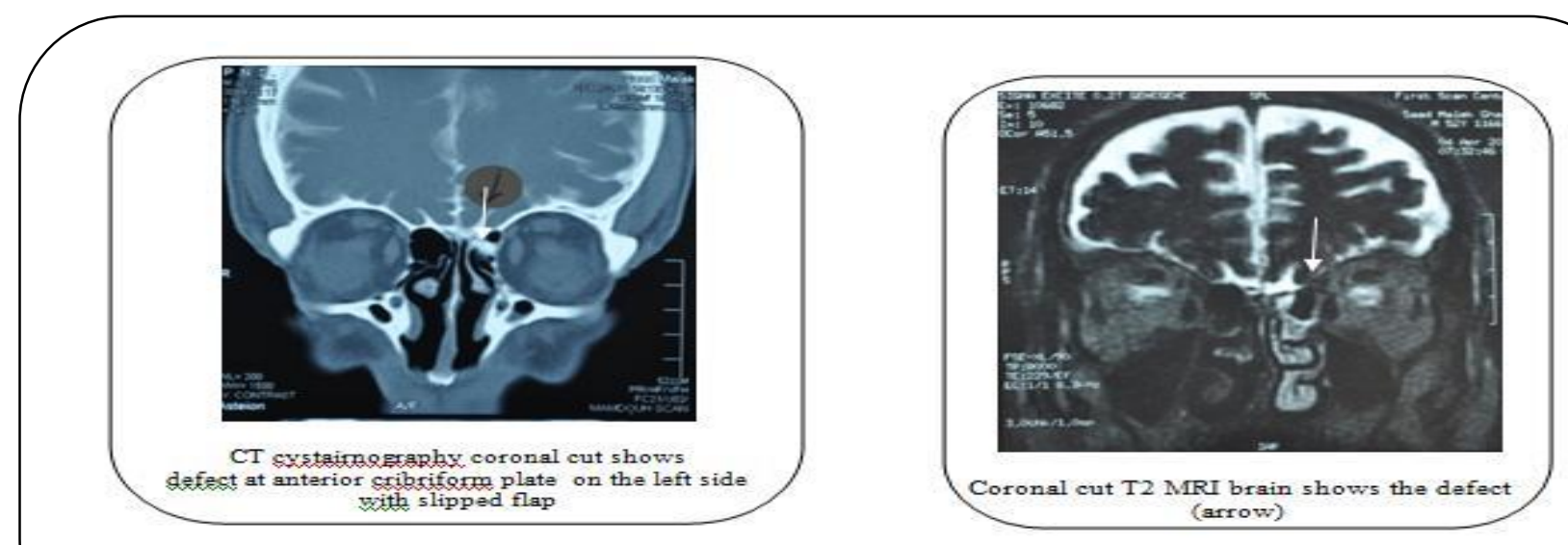

Fig. 6: showing preoperative imaging a case of spontanous CSF rhinorrhea
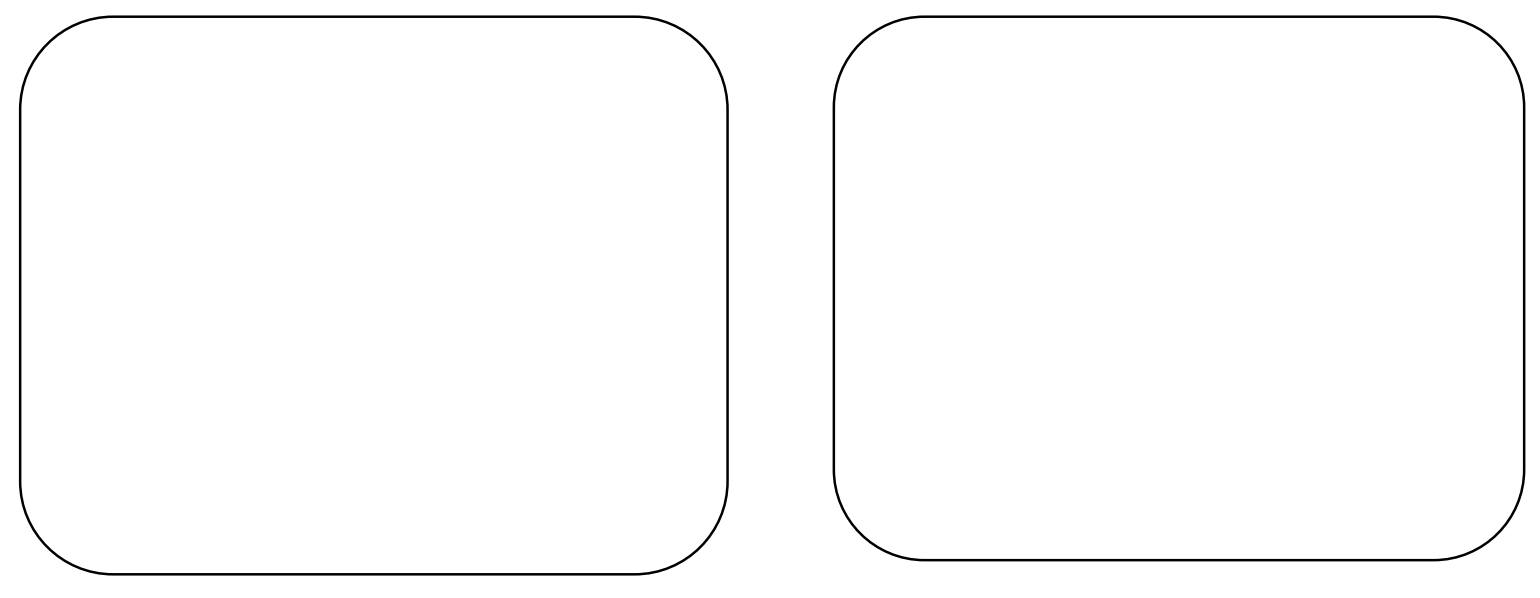
Sayed Siam et al.

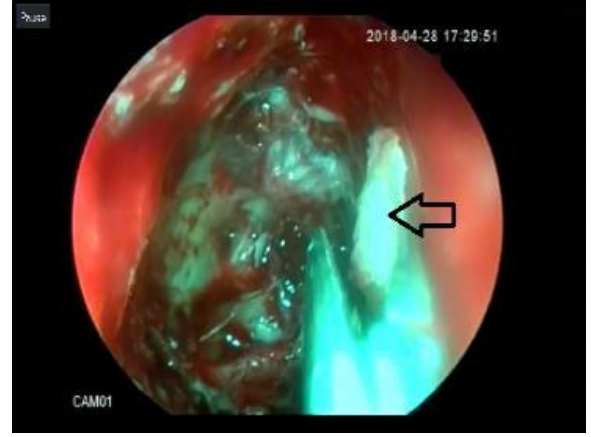

Figure 7: turbinate bone graft

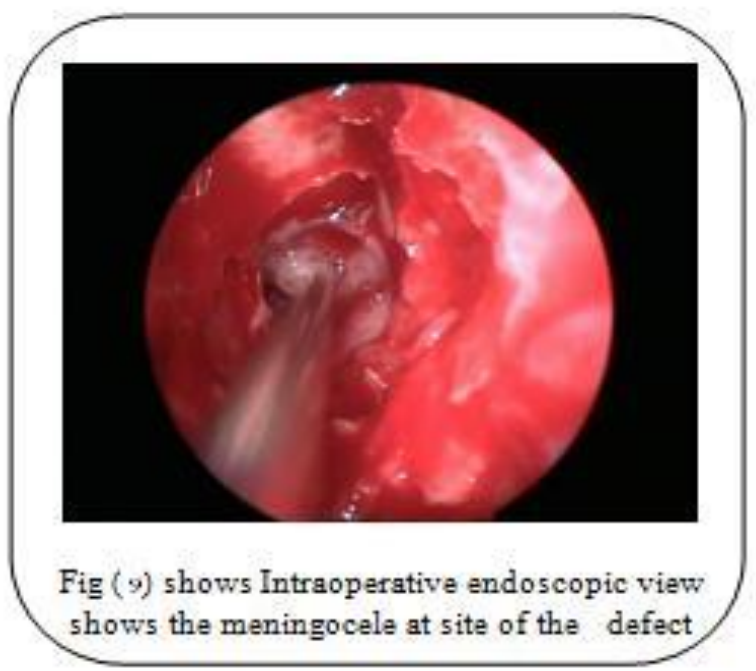

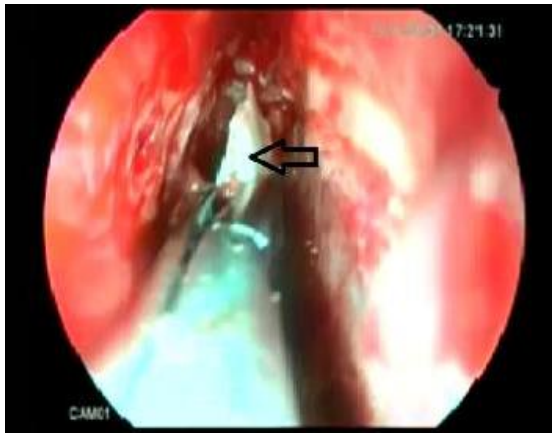

Figure 8: septal cartilage graft

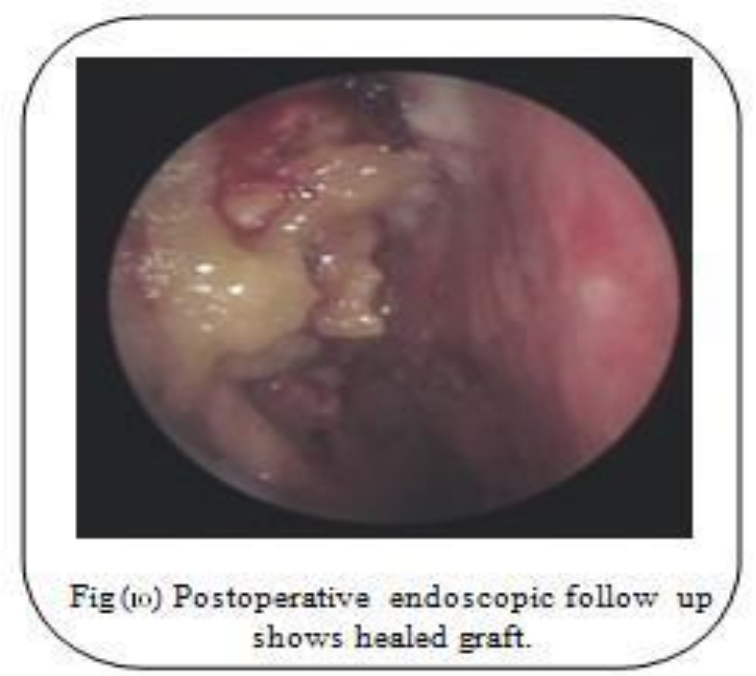

Table 8: showing pre-operative, operative and post-operative assessment of all cases of CSF leak

\section{Discussion:}




\begin{tabular}{|c|c|c|c|c|c|c|c|c|c|c|}
\hline Patient & 1 & 2 & 3 & 4 & 5 & 6 & 7 & 8 & 9 & 10 \\
\hline Sex & Male & Female & Female & Female & Male & female & Male & Female & Female & Male \\
\hline Age & 43 & 35 & 50 & 40 & 37 & 34 & 42 & 34 & 31 & 32 \\
\hline Atiology & Spontanous & $\begin{array}{l}\text { Spontaneou } \\
\text { s }\end{array}$ & $\begin{array}{l}\text { Surgical } \\
\text { trauma }\end{array}$ & Spontanous & $\begin{array}{l}\text { Spontaneou } \\
\mathrm{s}\end{array}$ & $\begin{array}{l}\text { Surgical } \\
\text { trauma }\end{array}$ & $\begin{array}{l}\text { Surgical } \\
\text { trauma }\end{array}$ & Spontanous & Spontanous & Spontanous \\
\hline Symptoms & $\begin{array}{l}\text { Watery } \\
\quad \text { nasal } \\
\text { Discharge } \\
\text { and } \\
\text { headach } \\
\end{array}$ & $\begin{array}{l}\text { Watery } \\
\text { nasal } \\
\text { discharge } \\
\text { and } \\
\text { headach } \\
\end{array}$ & $\begin{array}{c}\text { Watery } \\
\text { nasal } \\
\text { discharge }\end{array}$ & $\begin{array}{l}\text { Watery } \\
\text { nasal } \\
\text { discharge } \\
\text { and } \\
\text { headach }\end{array}$ & $\begin{array}{c}\text { Watery } \\
\text { nasal } \\
\text { discharge }\end{array}$ & $\begin{array}{l}\text { Watery } \\
\text { nasal } \\
\text { discharge } \\
\text { and } \\
\text { headach } \\
\end{array}$ & $\begin{array}{l}\text { Watery } \\
\text { nasal } \\
\text { discharge } \\
\text { and } \\
\text { headach } \\
\end{array}$ & $\begin{array}{c}\text { Watery } \\
\text { nasal } \\
\text { discharge }\end{array}$ & $\begin{array}{c}\text { Watery } \\
\text { nasal } \\
\text { Discharge }\end{array}$ & $\begin{array}{l}\text { Watery } \\
\text { nasal } \\
\text { discharge } \\
\text { and } \\
\text { headach } \\
\end{array}$ \\
\hline $\begin{array}{l}\text { Duration of } \\
\text { symptoms }\end{array}$ & 1 month & 4 months & 3 & $\begin{array}{ll}2 & \\
& \text { Months }\end{array}$ & 4 months & $7 \begin{array}{l} \\
\\
\end{array}$ & 3 Months & 1 Month & 20 days & $6 \begin{array}{l}6 \\
\text { months }\end{array}$ \\
\hline Side & Right & Right & Left & Right & Left & Right & Left & Left & Left & Right \\
\hline $\begin{array}{c}\text { Diagnosed } \\
\text { by }\end{array}$ & $\begin{array}{c}\text { CT Cystern- } \\
\text { graphy }\end{array}$ & $\begin{array}{c}\text { CT Cystern- } \\
\text { graphy }\end{array}$ & $\begin{array}{c}\text { CT Cystern- } \\
\text { graphy }\end{array}$ & $\begin{array}{c}\text { CT Cystern- } \\
\text { graphy }\end{array}$ & $\begin{array}{c}\text { CT Cystern- } \\
\text { graphy }\end{array}$ & $\begin{array}{c}\text { CT Cystern- } \\
\text { graphy }\end{array}$ & $\begin{array}{c}\text { CT Cystern- } \\
\text { graphy }\end{array}$ & $\begin{array}{l}\text { CT Cystern- } \\
\text { graphy }\end{array}$ & $\begin{array}{l}\text { CT Cystern- } \\
\text { graphy }\end{array}$ & $\begin{array}{c}\text { CT Cystern- } \\
\text { graphy }\end{array}$ \\
\hline $\begin{array}{l}\text { Site of } \\
\text { defect }\end{array}$ & $\begin{array}{l}\text { Rt. Cribrifo- } \\
\text { rm plate }\end{array}$ & $\begin{array}{l}\text { Rt. Cribrifo- } \\
\text { rm plate }\end{array}$ & $\begin{array}{l}\text { Lateral } \\
\text { wall of } \\
\text { sphenoid }\end{array}$ & $\begin{array}{l}\text { Rt. } \\
\text { Cribriform } \\
\text { plate }\end{array}$ & $\begin{array}{l}\text { Lt. Cribrifo- } \\
\text { rm plate }\end{array}$ & $\begin{array}{c}\text { Roof of } \\
\text { sphenoid }\end{array}$ & $\begin{array}{l}\text { posterior } \\
\text { wall of } \\
\text { sphenoid }\end{array}$ & $\begin{array}{l}\text { Lt fovea } \\
\text { ethmoidali s }\end{array}$ & $\begin{array}{l}\text { Lt. fovea } \\
\text { ethmoidali } \\
\mathrm{s}\end{array}$ & $\begin{array}{l}\text { Rt. Cribrifo- } \\
\text { rm plate }\end{array}$ \\
\hline $\begin{array}{l}\text { Size of the } \\
\text { Defect }\end{array}$ & $2 \mathrm{~mm}$ & $2 \mathrm{~mm}$ & $2,5 \mathrm{~mm}$ & $3 \mathrm{~mm}$ & $2 \mathrm{~mm}$ & $3 \mathrm{~mm}$ & $3 \mathrm{~mm}$ & $2,5 \mathrm{~mm}$ & $2 \mathrm{~mm}$ & $2,5 \mathrm{~mm}$ \\
\hline Graft used & $\begin{array}{l}\text { Septal } \\
\text { cartilage } \\
\text { graft and } \\
\text { mucosal } \\
\text { flab from } \\
\text { the septum }\end{array}$ & $\begin{array}{l}\text { Septal } \\
\text { cartilage } \\
\text { graft and } \\
\text { mucosal } \\
\text { flab from } \\
\text { the septum }\end{array}$ & $\begin{array}{l}\text { bone graft } \\
\text { of middle } \\
\text { turbinete } \\
\text { and fascia } \\
\text { lata graft }\end{array}$ & $\begin{array}{l}\text { Septal } \\
\text { cartilage } \\
\text { graft and } \\
\text { mucosal } \\
\text { flab from } \\
\text { the septum }\end{array}$ & $\begin{array}{l}\text { Septal } \\
\text { cartilage } \\
\text { graft and } \\
\text { mucosal } \\
\text { flab from } \\
\text { the septum }\end{array}$ & $\begin{array}{c}\begin{array}{c}\text { bone graft } \\
\text { of middle } \\
\text { turbinete } \\
\text { and fascial } \\
\text { lata graft }\end{array} \\
\end{array}$ & $\begin{array}{l}\text { bone graft } \\
\text { of middle } \\
\text { turbinete } \\
\text { and fascia } \\
\text { lata graft }\end{array}$ & $\begin{array}{l}\text { Septal } \\
\text { cartilage } \\
\text { graft and } \\
\text { mucosal } \\
\text { flab from } \\
\text { theseptum }\end{array}$ & $\begin{array}{l}\text { Septal } \\
\text { cartilage } \\
\text { graft } \\
\text { mucosal } \\
\text { from } \\
\text { septum }\end{array}$ & $\begin{array}{l}\text { Septal } \\
\text { cartilage } \\
\text { graft and } \\
\text { mucosal flab } \\
\text { from the } \\
\text { septum }\end{array}$ \\
\hline $\begin{array}{l}\text { Leak free } \\
\text { postope- } \\
\text { rative } \\
\text { Period } \\
\end{array}$ & 7 Months & 6 Months & 8 & $6 \begin{array}{ll}6 & \\
& \text { Months }\end{array}$ & $9 \begin{array}{ll}9 & \\
& \text { months }\end{array}$ & One year & $\begin{array}{ll}6 & \\
& \text { Months }\end{array}$ & 7 Months & $\mid \begin{array}{ll}9 & \\
& \text { Months }\end{array}$ & 6 Months \\
\hline Recurrence & NO & NO & NO & NO & NO & NO & NO & NO & NO & NO \\
\hline
\end{tabular}

Chronic rhinosinusitis with and without polyps:

In our study, $90 \%$ of the patients reported improvement in symptoms from surgery at the final follow up visit. $2(10 \%)$ patients were unchanged because of recurrence as on case was Samter's triad and another case was Kartegner's syndrome which have high recurrence rate. Our results compare very favorably to other studies of sinus surgery, Hoffman et al. reported about $90 \%$ improvement with mean follow up 9 months ${ }^{(10)}$. Kennedy reported $97.5 \%$ symptomatic improvement (11). Levine reported a success rate of $89 \%$ (12).

In agreement with results of Ramadan surgical causes of failure in patients with a previous endoscopic sinus surgery revealed that residual air cells and stenotic maxillary or frontal sinus Ostium were the most common causes. History of gastroesophageal reflux disease was predictor of poor symptomatic outcome with FESS. The mechanism by which GERD contributes to the pathogenesis of chronic sinusitis is not yet understood ${ }^{(13)}$.
Further study of the mechanism and relationship between GERD and chronic sinusitis may help us to understand this association ${ }^{(14)}$. In our series, two patients did not improve after surgery, because of recurrence of polyps in one case (Samter's triade) and the other case present by purulent discharge from ostia (kartenger's syndrome).

Stankiewicz suggested that the complications rate decreases with increasing experience, reporting a rate of $29 \%$ in the first 90 cases which he performed compared with only $2.2 \%$ in the subsequent 90 cases. Most of these complications were minor ${ }^{(15)}$. Kennedy reported no major complications in a series of 120 patients and few minor complications ${ }^{(11)}$. Gross et al. reported 14 minor complications and no major complications in their series which included 106 patients ${ }^{(16)}$. In our series, intraoperative complications were minor, only one case was injury to lamina paprycea. No severe postoperative haemorrhage which necessitated blood transfusion, no major complications was recorded postoperatively ${ }^{(16)}$. 


\section{Endoscopic treatment of fungal sinusitis}

In our study only one case was with allergic fungal sinusitis had recurrence after 6 months postoperative.

In the symptomatic assessment after the surgical procedure, we observed improvement in all items related with over $90 \%$ of the patients, confirming efficiency and benefit of the surgery, similar to the results obtained in patients of CRS ${ }^{(17)}$.

Using the same scale of values for symptoms, we observed significantly better symptomatic improvement in patients with fungal ball, who had fewer recurrences and required smaller number of reinterventions when compared to AFRS. These results evidenced the recurrent character and the severity of AFRS.

For patients with fungal ball, the level of recurrence (zero\%) is near to that found by Ferguson who estimated in the literature review as between $4 \%$ and $10 \%$. Manning and Kupfenberg considered that the recurrence of fungal ball is rare ${ }^{(18)}$.

The number of recurrences of AFRS was much greater, and in $33.3 \%$ of them they required surgical reintervention, marking the chronic and recurrent characteristic of the disease and its difficult control. The success of treatment with AFRS depends on three steps: to make surgical debridment to remove fungal antigens, allergic mucin and affected polypoid mucosa; to prevent recurrence of fungal growth, and to modify the immune response of host to antigen. Given that we do not always get the appropriate control of allergic symptomatology, we can expect postoperative follow-up with more recurrences.

\section{Septal and turbinate pathology}

Endoscopic septoplasty was described previously by another authors however, the techniques used have traditional septoplasty or sinus surgery instrumentation (19,20). According to Brennan et al. the ideal objective in septal surgery is permanent correction of deviation with avoidance of any complication. Four basic principles are consistent with this objective: good exposure; safe elevation of flaps; resection of only a limited, necessary amount of septum; and elimination of aetiological dynamic forces ${ }^{(21)}$.

However; Hwang et al. in their retrospective study of 111 patients undergoing endoscopic septoplasty, reported haematoma in $0.9 \%$, asymptomatic perforation in $0.9 \%$, and synechiae formation in $4.5 \%$ patients ${ }^{(21)}$.

In a retrospective study of 116 patients, Chung et al. described transient dental pain/hyperaesthesia in $4.3 \%$, asymptomatic septal perforation in $3.4 \%$, synechiae formation in $2.6 \%$, epistaxis 0.9 $\%$, septal haematoma in $0.9 \%$, and persistent septal deviation requiring revision septoplasty in $0.9 \%$ patients ${ }^{(22)}$.

However, in our study we reported only postoperative haemorrhage after removing of nasal packs (no blood transfusion) which occurred in one (10\%) patient operated for septoplasty with partial turbinectomy for which repacking was done for $24 \mathrm{~h}$. No patients had septal haematoma. One patient (10\%)had synechie formation, which was left alone which released by local anesthesia.

In our study, improvement in nasal obstruction was $100 \%$ and in headache was $(60 \%)$.This result is similar to results of Jain et al. in which improvement in obstruction was $96 \%$ and in headache was $40 \%{ }^{(23)}$.

\section{Endoscopic repair of spontaneous CSF rhinorhea}

The size of the defect appears to impact the surgical outcome, with larger defects likely to result in failure of repair and recurrent leaks. Composite osteomucosal or chondromucosal flaps have also been advocated for repair of defects greater than $1-2 \mathrm{~cm}$. Additionally, co-morbid conditions such as chronic cough may contribute to raised intracranial tension and failure of the repair ${ }^{(24)}$.

Presutti et al. in their 5-year retrospective study of 52 patients with endoscopic closure of CSF leak, used a septal mucoper- chondrial graft, with no lumbar drain and fluorescein tests. They reported a success rate of $88.5 \%$ on the first attempt $^{(25)}$.

Banks et al. in their 21-year retrospective study of 193 patients with 
endoscopic closure using intrathecal fluorescein localization of site of leak and lumbar drain in $73 \%$ had an initial success rate of $85-90 \%$ and an overall success rate of $98 \%{ }^{(26)}$.

Ye et al. in their 10-year retrospective study of 69 patients with no preoperative fluorescein injection, reported a success rate of $89 \%$ on the first attempt with an endoscopic multilayer reconstructive technique ${ }^{(27)}$.

Our results of endoscopic CSF rhinorrhea repair revealed a $100 \%$ success rate on the first attempt because the number of cases was small 1 (only 10 cases) and if the number of cases increase, complications and recurrence may be occur.

\section{CONCLUSION}

Functional endoscopic sinus surgery is the modern approach to surgery on the sinuses. In the past operations were designed to maximise drainage by gravity. As such large holes were fashioned into the sinuses and most of the lining tissue was removed. It is now known that this lining tissue plays a critical role in keeping sinuses healthy. As such the old-style surgery not infrequently worsened the problem.

Functional endoscopic sinus surgery places an emphasis on function. Given that we now know how important the normal anatomy and lining of the sinuses are to sinus health, the surgery is all about establishing ventilation and drainage along the normal pathways with maximum preservation of normal structures and in particular the sinus linings. The surgery is now a minimal rather than an extensive destructive procedure.

The availability of high definition cameras and very fine endoscopes allows for much greater control during the surgery and hence significantly better results.

\section{REFERENCES}

1. Varshney R, Frenkiel S, Nguyen LH, Youn M, Del Maestro R, Zeitouni A, Saad E and Funnell WR(2014): Simulator for endoscopic sinus surgery. $\mathbf{J}$.
Otolaryng. Head Neck Surg. , 43:40-46.

2. Slack $R$ and Bates $G$ (1998):

Functional endoscopic sinus surgery. American Family Physician, 58 (3): 707-718.

3. Cazzavillan A, Castelnuovo $\mathbf{P}$, Berlucchi M, Baiardini I, Franzetti A, Nicolai P, Gallo S and Passalacqua $G$ (2012): Pediatr allergy immunol.,22:32-44. doi: 10.1111/j.13993038.2012.01322..

4. Meccariello G, Deganello A, Choussy O, Gallo O, Vitali D, De Raucourt $D$ and Georgalas $C$ (2016): Endoscopic nasal versus open approach for the management of sinonasal adenocarcinoma. Head and Neck, 38 (1): 2267-2274.

5. Sukato DC, Abramowitz JM, Boruk M, Goldstein NA and Rosenfeld RM (2018): Endoscopic sinus surgery improves sleep quality in chronic rhinosinusitis. Otolaryngology-Head and Neck Surgery, 158 (2): 249-256.

6. Tajudeen BA and Kennedy DW (2017): Thirty years of endoscopic sinus surgery: what have we learned?. World Journal of Otorhinolaryngology - Head and Neck Surgery, 3 (2): 115-121.

7. Hirschmann A (1903): Endoscopy of the nose and its accessory sinuses. The Laryngoscope, 13 (10): 810-810.

8. Walter M (1978): Endoscopy of the Nose.: Urban and Schwarzenberg.

Baltimore,Maryland, 55:1-18

9. Kane KJ (2018): The early history and development of endoscopic sinonasal surgery in Australia (1985-2005). Australian Journal of Otolaryngology, 1:1.

10. Hoffman SR, Mahony M C, Chmiel J F et al. (1993): Symptom relief after endoscopic sinus surgery. Ear Nose Throat Journal, 72: 413-420.

11. Kennedy DW (1992): Prognostic factors outcomes and staging in 
ethmoid sinus surgery. Laryngoscope, 102:1-18.

12. Levine H L (1990): Functional endoscopic sinus surgery. Evaluation, surgery and follow up of 250 patients. Laryngoscope, 100: 79-84.

13. Ramadan H H (1999): Surgical causes of failure in endoscopic sinus surgery. laryngoscope, 109: 22-25.

14. Chambers D W, Davis D W, Cook P et al. (1997): Long term outcome analysis of functional endoscopic sinus surgery. Correlation of symptoms with endoscopic examination findings and potential prognostic variables. Laryngoscope, 107: 504-510.

15. Stankiewicz JA (1991): Cerebrospinal fluid fistula and endoscopic sinus surgery. Laryngoscope, 101:250-256

16. Gross R D, Sheridan $M F$ and Burgess L P (1997): Endoscopic sinus surgery complications in residency. laryngoscope, 107: 1080-1085.

17. Marple B, Newcomer M, Schwande $\mathbf{N}$ and Mabry $\mathbf{R}$ (2002): Natural history of allergic fungal rhinosinusitis. Otolaryngol. Head Neck Surg ., 127:361-316.

18. Manning SC, Merkel M, Kriesel K, Vuitch Fand Marple B (1997): Computed tomography and magnetic resonance diagnosis of allergical fungal sinusitis. Laryngoscope, 107:170-176.

19. Siegel N, Glicklich R, Taghizadeh $\mathrm{F}$ and Chang Y(2000): Outcomes of septoplasty. Otolaryngol .Head and Neck Surg., 122: 228-232.
20. Stammberger H (1991): Functional Endoscopic Sinosurgery. B.C. Decker, Philadelphia ,pp: 156-159.

21. Hwang PH, McLaughlin RB, Lanza DC and Kennedy DW (1999): Endoscopic septoplasty: indications, technique and results. Otolaryngol. Head Neck Surg., 120:678-682.

22. Chung BJ, Batra PS, Citardi MJ and Lanza DC(2007): Endoscopic septoplasty: revisitation of the technique, indications and outcomes. Am. J. Rhinol. , 21:307311

23. Jain Abramowitz JM., and Hawke M (2011): Essentials of Endoscopic Sinus Surgery. Edit. Robert Hurley, Mosby, Philadlephia.

24. Kelly TF, Stankiewicz JA, Chow JM, Origitano TC and Shea J (1996): Endoscopic closure of postsurgical anterior cranial fossa cerebrospinal fluid leaks. Neurosurgery, 106:1080-1083.

25. Presutti L, Mattioli F, Villari D, Marchioni $D$ and AlicandriCiufelli M (2009): Transnasal endoscopic treatment of cerebrospinal fluid leak. Acta Otorhinolaryngol. Ital., 29(4):191197.

26. Banks CA, Palmer JN, Chiu AG, O'Malley BW Jr, Woodworth BA and Kennedy DW (2009) :Endoscopic closure of CSF rhinorrhea. Otolaryngol Head Neck Surg., 140(6):826-833.

27. Ye H, Zuo J, Zhao H, Liu S, An $H$ and Liu Y (2010): Endonasal endoscopic repair of CSF rhinorrhea in a series of 69 patients. Br. J. Neurosurg ,24(3):244-248. 